Research Article

\title{
Overcoming Abdominal and Pelvic Cavity Workspace Constraints in Robotic-Assisted NOTES
}

\author{
Chung-Kwong Yeung $\mathbb{D},{ }^{1,2}$ Kwok-Wai Lam $\mathbb{D}^{2},{ }^{2}$ Jo Lai-Ken Cheung $\left.\mathbb{D}\right)^{2}$ \\ Adelina Tjokronegoro $\mathbb{D}^{2}{ }^{2}$ Chuk Shing Jones Law $\mathbb{D}^{2},{ }^{2}$ Satwinder Singh $\left(\mathbb{D},{ }^{2}\right.$ \\ Chi-Chung Foo $\mathbb{D}^{1},{ }^{1}$ Biji Sreedhar $\mathbb{1},{ }^{2}$ and Xuyen Dai Hoa $\mathbb{\circledR}^{2}$ \\ ${ }^{1}$ Department of Surgery, Li Ka Shing Faculty of Medicine, The University of Hong Kong, Pok Fu Lam, Hong Kong \\ ${ }^{2}$ Bio-Medical Engineering (HK) Limited, Cyberport, Hong Kong \\ Correspondence should be addressed to Chung-Kwong Yeung; ck.yeung@nisi.hk
}

Received 17 December 2019; Accepted 7 February 2020; Published 18 May 2020

Academic Editor: L. Fortuna

Copyright (c) 2020 Chung-Kwong Yeung et al. This is an open access article distributed under the Creative Commons Attribution License, which permits unrestricted use, distribution, and reproduction in any medium, provided the original work is properly cited.

\begin{abstract}
Despite early enthusiasm in robotic-assisted NOTES, several technical challenges exist. Various spinopelvic anatomical constraints can significantly act as obstacles and affect entry and space availability for the deployment of straight and rigid transvaginal/transanal NOTES instruments. Anatomical constraints such as the sacral slope, position, and distance to the target organs are defined. Transvaginal access to the surgical site required a high insertion angle between $20^{\circ}$ and $30^{\circ}$ to overcome the pronounced sacral slope resulting in dexterity and reachability limitations. A new set of robotic parameters was generated to introduce a 7 degrees of freedom robotic arm. Workspace simulation and phantom precision measurements have shown a significant improvement in the reachability and maneuverability of the robotic platform. While the robotic arms provided stable dexterity, it is constrained when reaching target sites in larger patients. This study has provided an insight and a solution in rigid instrument design, paving a safe route for transvaginal/transanal access for abdominal surgeries towards robotic-assisted NOTES.
\end{abstract}

\section{Introduction}

Today, over a million robotic-assisted operations had been performed using the da Vinci Surgical System, a preeminent commercially available surgical robotic system, with increasing popularity among surgeons in various extirpative and reconstructive procedures [1]. Robotic-assisted laparoscopic surgery has allowed surgeons to improve clinical outcomes by minimizing operative trauma, blood loss, infection rate, and shortening recovery time [2]. Laparo-endoscopic Single-site Surgery (LESS) and Natural Orifice Transluminal Endoscopic Surgery (NOTES) advances, these surgical procedures further in reducing invasiveness by minimizing or eliminating all incisions. LESS uses a single access port, usually with a subumbilical incision for the insertion of multiple instruments. In NOTES, access is gained through the body's own natural orifice such as the mouth, urethra, vagina, or anus. This method of access has brought substantial benefits to patients, including faster convalescence and therefore reduced hospital stay, minimized risks of wound infections, elimination of scars, and better cosmesis [3].

The development of NOTES for laparoscopic procedures was first carried out using flexible transgastric endoscopic tools for accessing the peritoneal cavity $[4,5]$. Since then, alternative access such as transoral, transurethral, transanal, or transvaginal routes has also been explored to perform surgical procedures [6]. One of the major technical difficulties in NOTES applications is in the use of flaccid instruments, which can create an unstable operative platform when manipulating solid organs from a distance. The lack of rigidity can also make force transmission and traction difficult, particularly when applying suturing and clipping [7]. NOTES can also share similar potential complications associated with LESS including overcrowding and collision of instruments, the inability to attain adequate triangulation, 
limited vision due to parallel alignment of instruments to the surgical site, and surgeon ergonomics [8].

As a result of these difficulties, robotic-assisted platforms for NOTES were developed. Robotic-assisted surgical platforms have shown greater potential in precision, dexterity, and surgeons' ergonomics. An earlier study demonstrated the feasibility of the da Vinci SiSystem in performing transvaginal NOTES nephrectomy in a cadaver model [9]. However, further studies conducted by different groups have suggested that suturing was difficult without the EndoWrist, clashing of external robotic arms and a lack in the workspace for bedside assistance also revealed major limitations $[10,11]$. In addition, the access to the peritoneal cavity requires all instruments to pass through the pelvic inlet that has a potential risk of injury to neighboring blood vessels or pelvic organs near the passage $[12,13]$.

Despite early enthusiasm of the scientific community to develop robotic-assisted NOTES and many having managed their way to preclinical studies, several technical challenges exist that remain to be addressed $[14,15]$. LESS and NOTES robotic designs are either linkage-based, wired-actuated, or continuum robots that are able to reach and overcome constraints within the narrow access orifice. Robots with a highly redundant degree of freedom are often considered for deployment into natural orifices (mouth, anus, and vagina); however, increased loads at the distal ends of long robotic manipulators can lead to potential distorted shapes of the robotic manipulator or instability in the position of the endeffector. Articulated designs with embedded actuation are proposed to address these issues. By design, articulated robotic arms with embedded actuators have a larger cross section that can make deployment difficult for NOTES. However, their rigid structure provides a stable platform to reach distant targets and operate under a larger load.

Recently, our group presented a novel surgical robotic system featuring a pair of robotic arms with 6 degrees of freedom (6-DOF) [16]. The aim was to develop a platform for robotic-assisted NOTES that can enable the performance of complex maneuvers in any surgical procedures. The system uses a 6-DOF external swivel mechanism as a stable platform and a cannula for the introduction of the robotic arms. The feasibility of the system was evaluated in performing a cholecystectomy procedure in a human cadaver for its application in transvaginal NOTES. However, it was identified when the sacral promontory was particularly prominent, the robotic arms could not effectively deploy and reach the target surgical site. Consequently, the cannula was inserted at a much steeper angle which inherently led the insertion angle of the robotic arms more than desired towards the anterior abdominal wall risking perforation. With the DOFs that were available in the robotic arms, the robotic arms were unable to reach the target surgical site.

Figure 1 below shows a sagittal view of a female abdominal-pelvic cavity. Major constraints in the spinopelvic regions are indicated at which the robotic arms were required to overcome before reaching into the abdominal cavity. Two routes of entry (vaginal and anal) are illustrated. The constraints on the spinal vertebrae will be similar in both cases. As shown on the image, the preparation of a safe

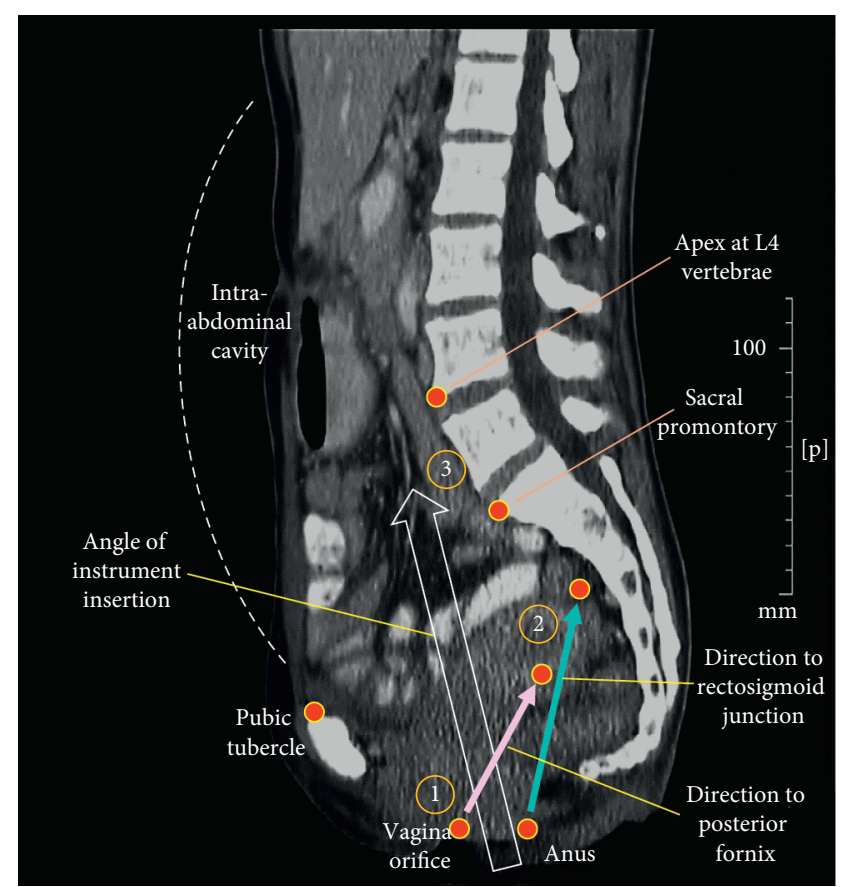

Figure 1: Routes for instrument placement for a transvaginal or transanal NOTES procedure. Location 1 indicates the access route from the vaginal orifice or the anal canal. Location 2 indicates the incision at the posterior fornix or the rectosigmoid junction at which the instruments needs to pass through. Location 3 indicates the spinal segments which the rigid instruments need to avoid.

access route requires a clear passage from location 1 at the entrance of the vaginal orifice to the posterior fornix or access through the anal canal towards an incision opening at the rectosigmoid junction (location 2). Unlike flexible devices, rigid laparoscopic or robotic instruments are required to be inserted at a slant angle anteriorly in a direction to prevent collision to the spinal segments as indicated by location 3.

A schematic of the bony obstacles and problematic instrument postures resulting from a high angle of instrument insertion overcoming a large sacral slope is shown in Figure 2. Three scenarios where the spinopelvic anatomy may pose problematic are illustrated:

Scenario 1. For patients with a large sacral slope and a prominent sacral promontory, a higher angle of instrument insertion $\beta$ is required to overcome the apex region located between lumbar vertebrae L3 to L4. This is crucial as the infrarenal aorta and inferior vena cava run parallel anterior to these spinal segments. Placement of instruments should also avoid causing a possible risk of vascular and bowel injuries within the peritoneal cavity as exemplified by location 1 in Figure 2.

Scenario 2. In cases where a large angle of instrument insertion $\beta$ is required, the insertion of the robotic arms may hit the anterior abdominal wall as illustrated by location 2 in Figure 2. 


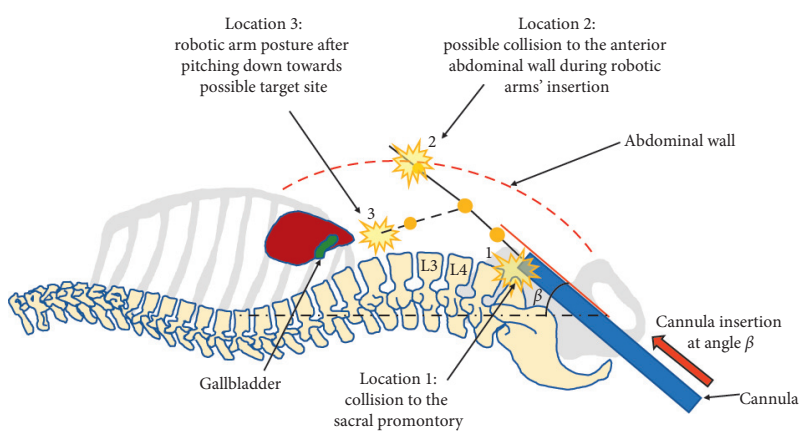

Figure 2: Possible collision sites for NOTES deployment.

Scenario 3. In another possible setting, the robotic arms cannot reach the target site due to the length and joint limits of a robot design as illustrated by location 3 in Figure 2. The work plane of the manipulator in this scenario has also been displaced anteriorly away from the intended target organ that would limit the reachability and dexterity in manipulation for a surgical operation.

The objectives of this study were to define the anatomical constraints in a transvaginal NOTES surgery and their impact on the design of a surgical robotic system. According to the EURO-NOTES clinical registry, approximately $85 \%$ of surgical procedures performed were transvaginal cholecystectomy [14], and therefore the reachability of the gallbladder was selected as a model in the current study. A new fully internally motorised rigid robotic arm design is presented that introduces an additional DOF at the elbow joint to provide greater flexibility to the surgical robotic system to conduct abdominal and pelvic surgeries using a transvaginal/transanal NOTES approach. Design considerations include new joint kinematics introduced to prevent collisions with the abdominal wall and, at the same time, dexterous robotic arms with sufficient reachability and precision after its deployment when anatomical constraints such as a large sacral slope are encountered.

\section{Materials and Methods}

2.1. Anatomical and Design Constraints for Robotic Arm. The present study formulates a robotic platform for NOTES application based on a common procedure of conducting a transvaginal cholecystectomy. In a study that was conducted in Japanese women, an average distance to the gallbladder measured from the posterior vagina fornix was $26.1 \mathrm{~cm}$. The shortest distance was recorded at $19.8 \mathrm{~cm}$ and the furthest distance at $32.2 \mathrm{~cm}$ [17]. No similar data was found among the Caucasian population at the time of the literature review. In a German study, the distance for long manual instruments to reach the gallbladder through the vaginal orifice was reported to be approximately $40 \mathrm{~cm}$ [18]. Assuming this instrument length is representative and taking into account the length of the vaginal canal to be between $4 \mathrm{~cm}$ and $13 \mathrm{~cm}$ as reported from a European study [19], the distance of the gallbladder from the vaginal fornix was estimated to be in a range of $27 \mathrm{~cm}$ to $36 \mathrm{~cm}$ among females in a Caucasian population. The approximate size of the gallbladder is estimated as $7-10 \mathrm{~cm}$ in length and about $4 \mathrm{~cm}$ in diameter. An assumption was made with this volume of the gallbladder as the target zone where the end-effector of the robotic arms must reach. Two target zones of the gallbladder were taken based on the nearest distance of $19.8 \mathrm{~cm}$ from the Japanese study and the furthest distance of $36 \mathrm{~cm}$ from the European study.

2.2. Design of a Robotic System. The robotic arms were designed to mimic the mobility of a surgeon's shoulder, arm, and wrist for performing abdominal and pelvic surgical procedures. The robotic arms are incorporated into a telemaster-slave system, consisting of robotic arms, a cannula that is fitted with an air seal mechanism, an HD stereoscopic camera, and a bedside mounting swivel. The latter can position and fix the cannula in three directions (telescopic, pitch, and sway) and orientations to provide reachability of the deployed robotic arms within the abdominal cavity.

Upon the insertion of the cannula, each robotic arm is inserted and anchored away from the central lumen of the cannula. A camera on a tentacle arm is similarly inserted and secured, leaving the lumen of the cannula free for the insertion of additional surgical tools, gauze, or for tissue retraction. Each robotic arm is $17.1 \mathrm{~cm}$ in length from the shoulder joint to the wrist joint (excluding the length of the end-effector) with a diameter of $16 \mathrm{~mm}$.

To overcome anatomical constraints of a pronounced sacral promontory, provide optimized triangulation, maximize reachability, and provide positional stability under payload, the robotic arms were designed with an extra degree of freedom. The DOFs consist of 2-DOFs at the shoulder and 2-DOFs at the elbow to provide optimized triangulation, followed by 3-DOFs at the wrist required for orientation of end-effector. The 7-DOFs are internally motorised along the length of the robotic arms, with an additional motor to actuate the end-effector, e.g., grasper.

Seven inputs are encoded by the telemaster controller manipulators including the position $(x, y, z)$, rotations $(R x$, $R y, R z)$, and elbow level $(h)$. As a result, the robotic arms are fully constrained and an inverse kinematic solution can be derived. To ensure real-time operation, the three wrist joints are assumed to be intersecting for an analytical solution. The resultant translational error of the last joint is found and adjusted for using the forward kinematics.

Figure 3 and Table 1 provide the description of the D-H parameters of the 7-DOF robotic arm design. The range of joint angles $\theta_{i}$, at each DOF is indicated in Figure 3.

Based on the $\mathrm{D}-\mathrm{H}$ parameters and joint limits, the reachable envelop of the robotic armwas defined by an outer boundary (full extension of the robotic arms) and inner boundary (elbow joints are at their limits), as shown in Figures 4(a) and 4(b)). The shared workspace's height, width, and depth were determined as $288 \mathrm{~mm}, 80 \mathrm{~mm}$, and $267 \mathrm{~mm}$, respectively. The effective shared workspace that is reachable by both arms has been calculated to be under $40 \%$ to the overall working envelop of both arms (Figure 4(c)). 


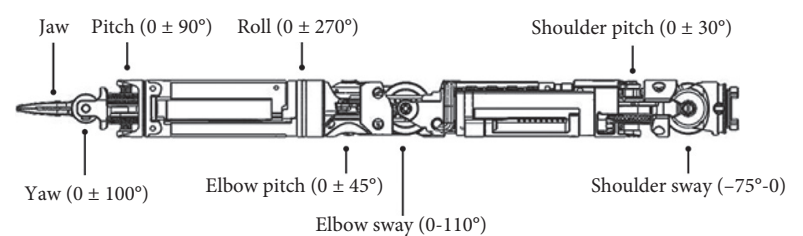

Figure 3: Design of the 7-DOF robotic arm.

TABle 1: D-H parameters of the proposed new design of 7-DOF robotic arm for NOTES.

\begin{tabular}{|c|c|c|c|c|c|}
\hline Link & $a_{i-1}(\mathrm{~mm})$ & $\alpha_{i-1}(\operatorname{deg})$ & $d_{i}(\mathrm{~mm})$ & $\theta_{\mathrm{i}}(\mathrm{deg})$ & DOF \\
\hline 1 & 0 & 0 & 0 & $\theta_{1}$ & Shoulder sway \\
\hline 2 & 16.5 & $-90^{\circ}$ & 0 & $\theta_{2}$ & $\begin{array}{c}\text { Shoulder } \\
\text { pitch }\end{array}$ \\
\hline 3 & 65.5 & $90^{\circ}$ & 0 & $\theta_{3}$ & Elbow sway \\
\hline 4 & 16.5 & $-90^{\circ}$ & 0 & $\theta_{4}$ & Elbow pitch \\
\hline 5 & 0 & $90^{\circ}$ & 64.6 & $\theta_{5}$ & Roll \\
\hline 6 & 0 & $90^{\circ}$ & 0 & $\theta_{6}$ & Pitch \\
\hline 7 & 11.7 & $-90^{\circ}$ & 0 & $\theta_{7}$ & Yaw \\
\hline
\end{tabular}

2.3. Target Reachability and End-Effector Displacement Stability. The positional accuracy of the robotic arm was measured with an optical tracking system (NDI, Polaris Vega, Canada), to provide the relative displacement of the robotic arm with respect to the master control manipulators. Measurements were taken in the horizontal ( $x$-axis), vertical $(y$-axis), and telescopic ( $z$-axis) directions. RMS errors were calculated and normalized to the manipulation displacements. Accuracy and reachability were determined by deployiment of the robotic arms and cannula into a $3 \mathrm{D}$ printed model phantom. A touchscreen was placed within the abdominal cavity, simulating the location of the gallbladder, and the touchpoints of the end-effector were recorded. The touchscreen (3.5 inch RPi Display XPT2046, $320 \times 480$ resolution, with Raspberry Pi $3 b+$ controller), has a spatial resolution of $0.0154 \mathrm{~mm}$ per pixel.

2.4. Surgical Tasks. Surgical tasks were modelled using a dexterity skill set (\#4127/\#4008, Chamberlain Group, UK), including direct end-effector-to-end-effector and "wirerollercoaster" peg transfers. The tasks were repeated 6 times and an average time was reported.

\section{Results}

3.1. End-Effector Displacement and Reachability for Cholecystectomy. The base of the robotic platform inside the abdominal cavity was carefully located in order to maintain the intended workspace for the deployment of the robotic arms to form proper triangulation and reachability in a surgical operation. The base of the robotic platform can be displaced by adjusting the position of the cannula when both robotic arms were securely anchored at its distal end. The orientation of the cannula was crucial to overcome anatomical obstacles according to findings in the current study. A rigid 6DOF swivel system provided a stable anchor to the platform and positioning of the cannula with translational and rotational motions along the $x, y$, and $z$ axes. A torso phantom model, which was designed based on validated data sets from human CT scan images, was adapted to create simulations in the physical intra-abdominal workspace [20]. Based on this model, the data set was rescaled and adjusted using local data of a female patient with a sacral slope of $45^{\circ}$ and a distance of $21 \mathrm{~cm}$ between the posterior vaginal fornix to the gallbladder. A resin torso phantom model using this modified data set was built by a selective laser sintering (SLS) rapid prototyping method with the removal of the abdominal wall such that deployment and manipulations of the new 7-DOF robotic platform could be observed. Figure 5 shows the image of the female phantom model with the cannula insertion angle of $30^{\circ}$ and successful deployment of the new robotic arms. Direct end-effector-to-end-effector and "wire-rollercoaster" peg transfers were completed with an average transfer time of $1.08 \mathrm{~min}$ and $2.59 \mathrm{~min}$, respectively.

The robotic arms were controlled by a master-slave system with the robotic arm motion mapped to the master hand controller position at $50 \mathrm{~Hz}$ intervals. Figure 6 shows the root mean square (RMS) error between the displacement of the hand controller and the robotic arm end-effector as the hand controller is independently moved in a horizontal, vertical, and telescopic direction $(x, y, z)$. The measured errors arise from parasitic movements in the other orthogonal planes as the end-effector moves in a given direction. Errors were also attributed to the approximate iterative reverse kinematic solutions and lag time between the controller and end-effectors. Errors were measured as the end-effector wrist joint was moved from a stationary position to a travel distance of $5 \mathrm{~cm}$, held in position for $5 \mathrm{sec}$ and returned to its original starting point. RMS errors were within $10 \%(5 \mathrm{~mm})$ in all three directions.

In a deployed position, targeting transvaginal access to the gallbladder, the additional degree of freedom at the elbow allows the forearms to pitch downward when a large insertion angle is required to overcome a pronounced sacral promontory. At an elbow pitch angle of $20^{\circ}$ downward, the displacement tracking wasmaintained comparable to the elbow pitch angle of $0^{\circ}$ (Figure 7), providing a stable triangulation posture.

While the initial design sought to develop a NOTES deployable robotic system for a wide target population with and without a pronounced sacral promontory, it was found to be difficult to achieve. Given the wide range of anatomical variations between the Asian and Caucasian female population, our initial investigation suggests that a single arm design would not have sufficient length to reach the gallbladder for both populations. For an Asian population with an average and shortest distance to the gallbladder measured from the posterior vagina fornix was $26.1 \mathrm{~cm}$ and $19.8 \mathrm{~cm}$, respectively, the robotic system can reach the target site. Based on the scaled phantom model with an assumed transvaginal insertion, target points A-F were registered to encompass the surgical field within the upper abdominal region. Target points $\mathrm{B}$ and $\mathrm{E}$ represent the upper and lower limits, respectively, along the median plane, points $\mathrm{A}, \mathrm{E}$ and points $\mathrm{C}, \mathrm{F}$ represent the insertion of the instrument with an angle of deviation of $-20^{\circ}$ and $+20^{\circ}$, respectively, lateral to the medial axis (Figure 8). 


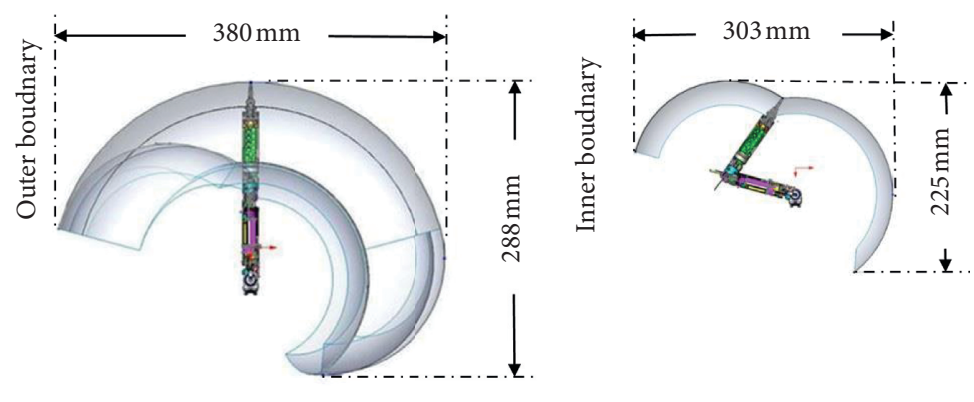

(a) (b)

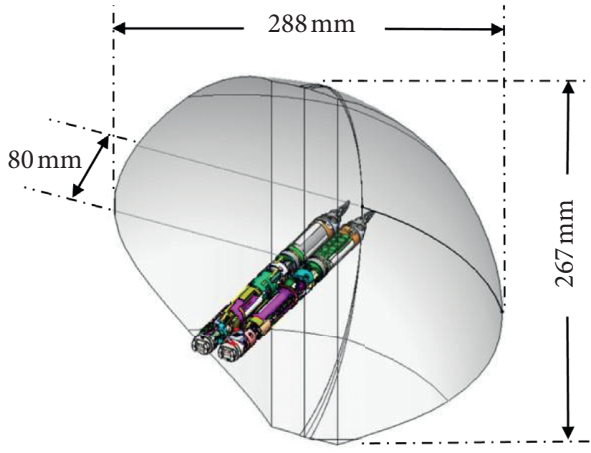

(c)

FIGURE 4: Robotic arm (a) outer workspace limits, (b) inner workspace limit, and (c) shared workspace.

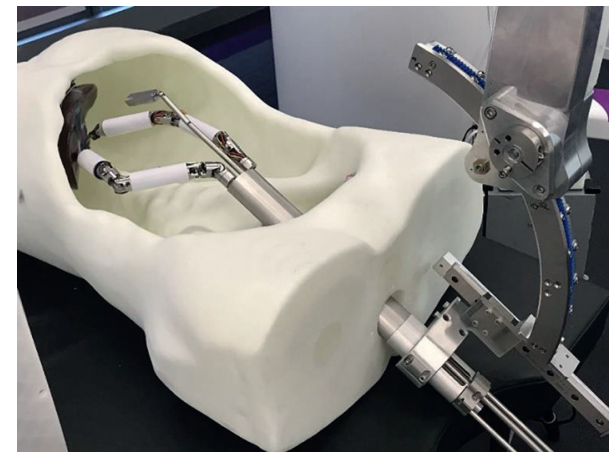

FIGURE 5: Robotic arm and cannula deployment in a female phantom model.

Accommodating for a range in the angle of the sacral slope, the insertion cannula was inclined (angle $\beta$ ) to range from $10^{\circ}$ to $30^{\circ}$ from the horizontal plane of the patient bed, as well as $\pm 20^{\circ}$ laterally from the medial plane. The reachability and accuracy were measured at the target points $\mathrm{A}-\mathrm{F}$, where the longest distance from the vaginal orifice to points $\mathrm{A}-\mathrm{F}$ include the average length of the gallbladder $(8.5 \mathrm{~cm})$, and points D-F to simulate the shortest distance. With the masterslave control, the robotic arm can reach the target accurately, within $0.2 \mathrm{~mm}$. At an insertion angle of $25^{\circ}$, the robot arms are able to reach the distal points $(\mathrm{A}-\mathrm{C})$; however, joint limits prevent the arms from reaching closer targets (D-F) with proper triangulation. At high insertion angles ( $30^{\circ}$ and above), reachability of distal target is problematic with the current design unable to reach points $\mathrm{A}, \mathrm{B}$, and $\mathrm{C}$ (Figure 8). At these high insertion angles, the elbow pitch and robotic forearm length cannot overcome the vertical distance to reach the target. While the cannula could be inserted further inward. However, the apex of the robotic arm can collide with the upper abdominal wall and require a larger shoulder, elbow pitch angle, or longer robotic arm lengths that are not possible in the current configuration of the system.

\section{Discussion}

In transvaginal NOTES surgeries, instruments are inserted into the vaginal canal and through an incision at the posterior vaginal fornix to gain access into the abdominal cavity.
Various anatomical constraints can significantly act as obstacles and affect entry and space availability for the deployment of transvaginal instruments. These constraints may include organs within the pelvic cavity and bony structures like the lower lumbar vertebrae and the sacral promontory. The deployment and maneuverability of surgical instruments for NOTES can be very confined and technically challenging.

The introduction of an extra DOF to the robotic arm was designed to enhance its reachability to organs in situations where a difficult anatomical constraint such as a prominent sacral promontory can pose problems. A workspace study was constructed under different anatomical constraint assumptions, which includes a lordotic condition where a sacral slope larger than $35^{\circ}$ and a bony prominence located at L3 to L4 lumbar vertebrae was present: a tight restriction of the cannula orientation due to ligamentous constraints within the pelvic cavity and a distant target organ in the abdominal cavity from the vaginal orifice. In this simulation, with reference to Nakajima's study, a negative angle was assumed to examine a more posterior position of the gallbladder along the anterior-posterior direction.

A classification system proposed by Vaz et al. characterize the human spinopelvic posture into four types based on the variation in the sagittal alignments between the association of the lumbar spine and the pelvis [21]. One of the determining pelvic parameters in this association is the sacral slope, which is the angle between the superior endplate of the first sacral bone to the vertical axis. A large sacral slope has a close relationship in individuals with a lordotic lumbar vertebrae. Lordosis indicates an excessive inward curvature in the lumbar vertebrae, which gives rise to a more prominent sacral promontory and can present as a major constraint for access into the abdominal cavity for rigid instruments, particularly when transvaginal/transanal access is considered. In one prospective study on sagittal morphology in healthy volunteers, no statistical difference between males and females was identified, but a significant correlation between lumbar lordosis and the sacral slope was observed [21]. Published data have shown that up to $63 \%$ Asian and $68 \%$ Caucasian adult population fall under type 3 and type 4 lumbar lordosis with a sacral slope over $35^{\circ}$ and with a bony prominence observed between L3 to L4 lumbar vertebrae [22, 23]. In this regard, 

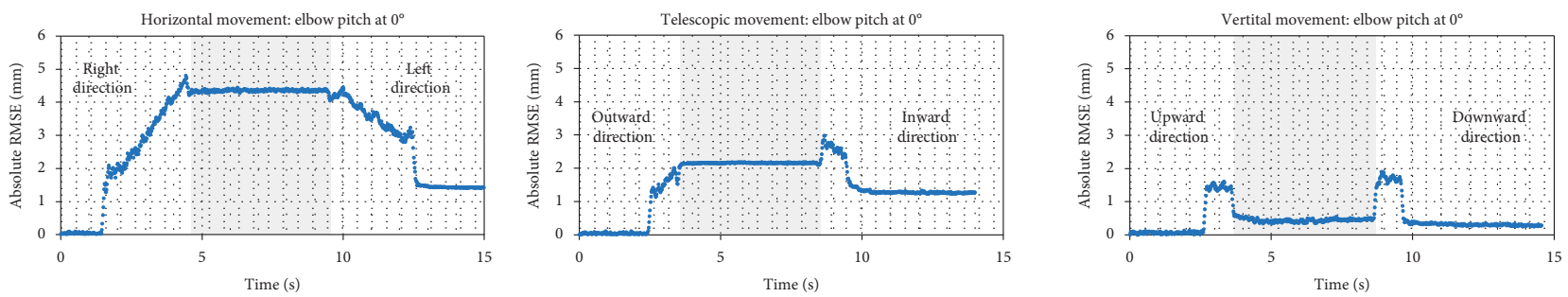

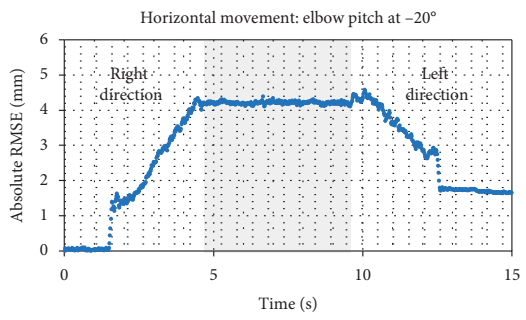

(a)

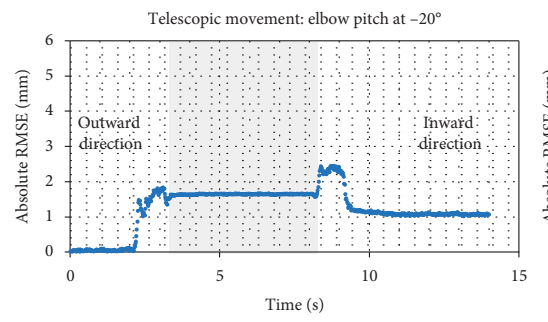

(b)

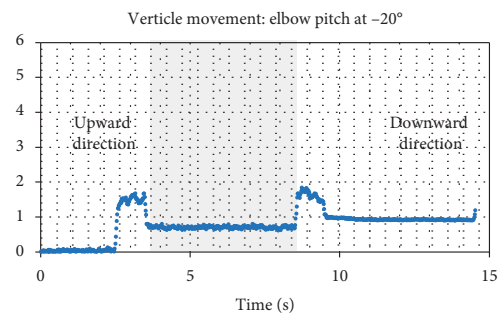

(c)

FIgURE 6: RMS error in vertical, horizontal, and telescopic directions due to parasitic movements.

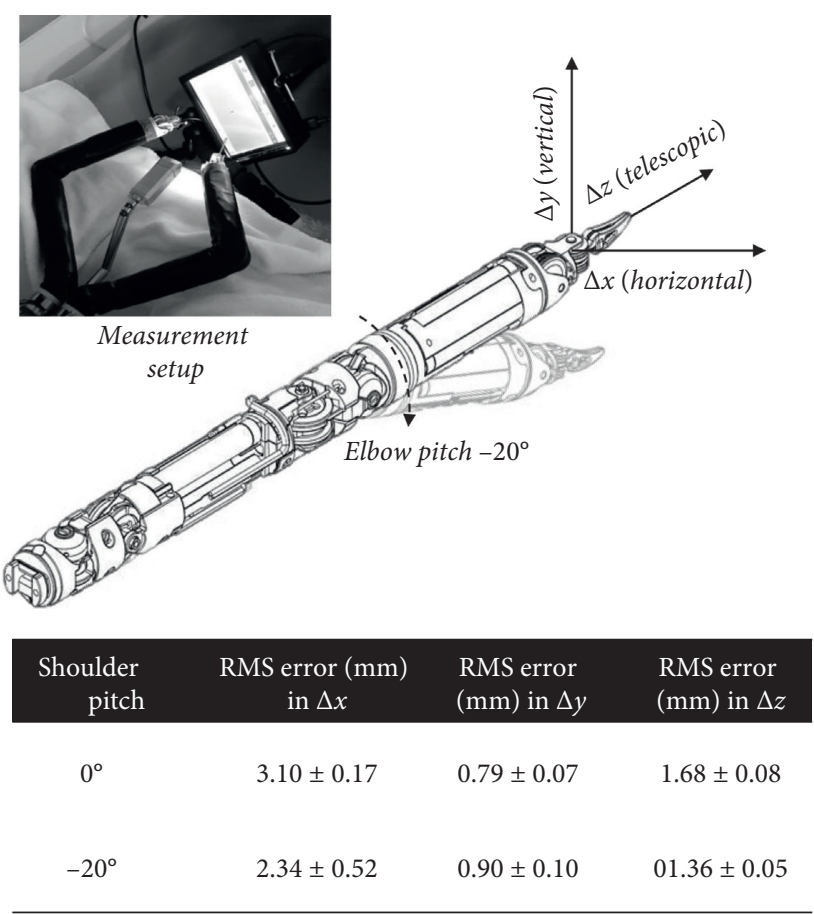

FIGURE 7: Average RMS error in three orthogonal directions with robotic arm elbow pitch at $0^{\circ}$ and $-20^{\circ}$.

over half of the population would have a large sacral slope and their bony prominence would potentially form major obstacles during transvaginal NOTES.

The sacral promontory will not be a direct obstacle to the robotic arms' deployment but will affect the angle of insertion from the cannula that can shift the work plane anteriorly, resulting in a deficiency in the ability of the robotic arms to form triangulation within a target region. After fixing the base position of the robotic arms by a steeper angle of cannula insertion to overcome the protruded sacral promontory and lumbar vertebrae, the effect from the displaced work plane of the whole robotic platform will be minimized by the robotic arms that are configured with both the shoulder pitch joint and an additional elbow pitch joint. Such an arrangement will significantly increase the flexibility for the robotic arms deployment. Combining the shoulder and elbow yaw joints can allow the robotic arms to form optimum triangulation within the region at the position of the gallbladder. The cannula was utilized to overcome obstacles posed by the prominent sacral promontory. Based on our model, a required angle of cannula insertion was determined to be between $20^{\circ}$ and $30^{\circ}$ from a horizontal plane assuming a sacral slope of $45^{\circ}$ at the $3 \mathrm{D}$ lumbar spine and pelvis models that simulate a patient with type 3 or type 4 lordotic condition in a lithotomy position. This angle of cannula insertion is crucial as the first step for a successful robotic-assisted NOTES in order to prevent a collision to bony obstacles and any injury to adjacent tissue or important blood vessels during the insertion of robot arms.

Based on the above-mentioned assumptions, the posture of the 7-DOF robotic arms to reach the gallbladder was simulated at the nearest distance of $19.8 \mathrm{~cm}$, respectively, from the vaginal orifice. However, at a farther distance of $28.3 \mathrm{~cm}$, the robotic arms can reach the target site but lose its triangulation posture and dexterity. Figure 9 illustrates the virtual workspace simulation in the deployment of the robotic platform at different longitudinal positions within the intra-abdominal cavity.

In the Asian population with a pronounced sacral and type 3-4 lordotic conditions, the robotic arms have demonstrated its reachability using a phantom model while maintaining stable triangulation and accuracy with $0.2 \mathrm{~mm}$, noting that the maximum insertion angle of $25^{\circ}$ is possible. With the Caucasian population and larger Asian women, the larger distance between the vagina fornix and the gallbladder cannot be overcome with the current robotic arm kinematic design, despite the additional DOFs prescribed by the swivel system. The large distance can be overcome with a cannula further inserted towards the gallbladder; however, with a $20^{\circ}$ 


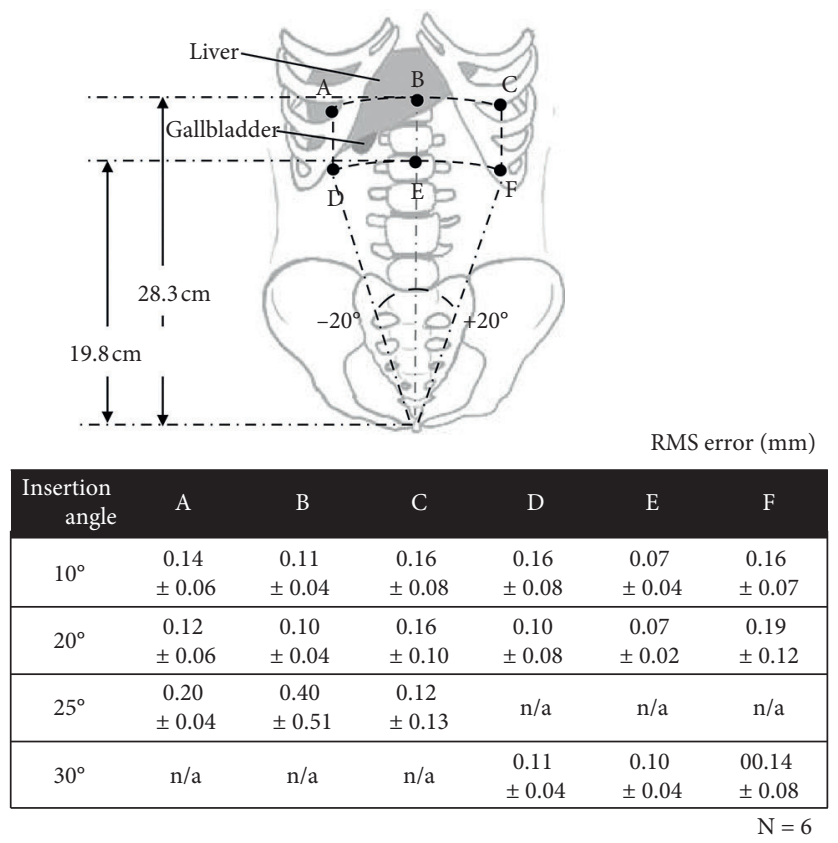

FIgURE 8: Reachability with measured RMS error at multiple cannula insertion angles with a maximum insertion angle of $25^{\circ}$ for distal points $\mathrm{A}, \mathrm{B}, \mathrm{C}$, and $30^{\circ}$ for proximal points $\left.\mathrm{D}, \mathrm{E}, \mathrm{F}\right)$.

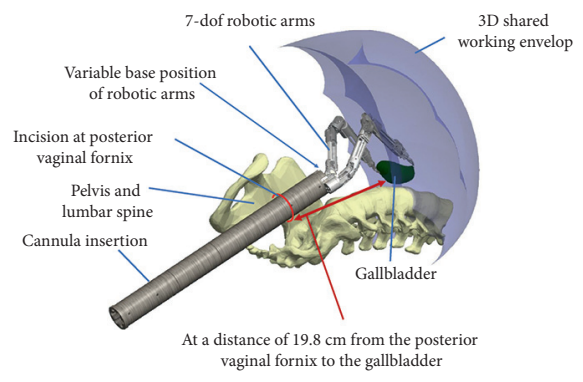

(a)

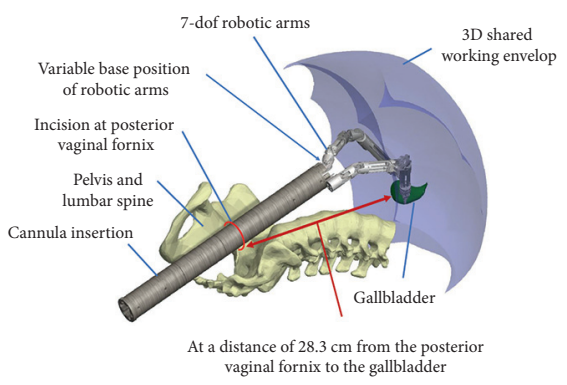

(b)

FIGURE 9: Workspace overlap and reachability with gallbladder at (a) $19.8 \mathrm{~cm}$ while maintaining end-effector dexterity and (b) $28.3 \mathrm{~cm}$ where dexterity is lost.

to $30^{\circ}$ cannula insertion angle, telescopic insertion may cause the robotic arm to collide with the abdominal wall. This further increases the lateral (vertical) distance between the anchor and the target requiring the robotic arms to be deployed at its kinematic limits and thus losing most of its dexterity. Further optimization and customization of the robotic arms are required to extend the reachability of the system for cases with larger anatomical features. A collision avoidance mechanism would be critical in the kinematic control system. In future work, the integration of tactile sensors could provide additional inputs to improve the robotic controls $[24,25]$.

This study has shown a promising direction in the design of a robotic platform that can accommodate variations of anatomical constraints towards a NOTES procedure. Moreover, a proper preoperative image assessment in lordotic patients with prominent bony pelvic anatomy is important for a NOTES procedure whether in a transvaginal or transanal approach, particularly when rigid instruments or robotic platform is used. This will potentially minimize intraoperative risks and benefit patients with the advantages of NOTES.

\section{Conclusions}

The development of instrumentation for a NOTES procedure is vigorous worldwide, but at present, a promising solution is still in demand to improve the technique and standardization of instruments. In this study, we have designed and introduced an additional pitch at the elbow joint in a robotic platform that allows the arms to overcome anatomical constraints such as the prominent sacral promontory and yet to contain a large and dexterous shared workspace. While the study of current design 7-DOF robotic arms points to the need to customize the robotic kinematic parameter for variation in distance to reach the target site, this design is a step forward towards pure NOTES, especially 
through transvaginal access. Further work is warranted to evaluate its performance in preclinical studies.

\section{Data Availability}

The data used to support the findings of this study are available from the corresponding author upon request.

\section{Conflicts of Interest}

Chung-Kwong Yeung is the Chairman and CTO of BioMedical Engineering (HK) Limited. Adelina Tjokronegoro, Chuk Shing Jones Law, Kwok-Wai Lam, Jo Lai-Ken Cheung, Biji Sreedhar, and Xuyen Dai Hoa are employees of BioMedical Engineering (HK) Limited. Satwinder Singh was a past employee at Bio-Medical Engineering (HK) Limited. Chi-Chung Foo has no conflicts of interest.

\section{Acknowledgments}

The study was supported in part by the funding from BioMedical Engineering $(\mathrm{HK})$ Limited and the Innovation and Technology Commission of HKSAR (project no. ITS/149/13FX).

\section{References}

[1] Intuitive Surgical Inc., Investor Presentation Q4, Intuitive Surgical Inc., Sunnyvale, CA, USA, 2019.

[2] J. E. Anderson, D. C. Chang, J. K. Parsons, and M. A. Talamini, "The first national examination of outcomes and trends in robotic surgery in the United States," Journal of the American College of Surgeons, vol. 215, no. 1, pp. 107-114, 2012.

[3] D. N. Moris, K. J. Bramis, E. I. Mantonakis, E. L. Papalampros, A. S. Petrou, and A. E. Papalampros, "Surgery via natural orifices in human beings: yesterday, today, tomorrow," The American Journal of Surgery, vol. 204, no. 1, pp. 93-102, 2012.

[4] A. N. Kalloo, V. K. Singh, S. B. Jagannath et al., "Flexible transgastric peritoneoscopy: a novel approach to diagnostic and therapeutic interventions in the peritoneal cavity," Gastrointestinal Endoscopy, vol. 60, no. 1, pp. 114-117, 2004.

[5] P.-O. Park, M. Bergström, K. Ikeda, A. Fritscher-Ravens, and P. Swain, "Experimental studies of transgastric gallbladder surgery: cholecystectomy and cholecystogastric anastomosis (videos)," Gastrointestinal Endoscopy, vol. 61, no. 4, pp. 601-606, 2005.

[6] S. A. Antoniou, D. K. Bartsch, and D. K. Bartsch, "NOTES: current status and recent developments," Viszeralmedizin, vol. 28, no. 6, pp. 388-394, 2012.

[7] S. Zuo and S. Wang, "Current and emerging robotic assisted intervention for Notes," Expert Review of Medical Devices, vol. 13, no. 12, pp. 1095-1105, 2016.

[8] E. D. Flora, T. G. Wilson, I. J. Martin, N. A. O’Rourke, and G. J. Maddern, "A review of natural orifice translumenal endoscopic surgery (NOTES) for intra-abdominal surgery," Annals of Surgery, vol. 247, no. 4, pp. 583-602, 2008.

[9] H. Laydner, R. Autorino, W. Isac et al., "Robotic retroperitoneal transvaginal natural orifice translumenal endoscopic surgery (NOTES) nephrectomy: feasibility study in a cadaver model," Urology, vol. 81, no. 6, pp. 1232-1238, 2013.

[10] S. Micali, G. Pini, D. Teber et al., "New trends in minimally invasive urological surgery. What is beyond the robot?" World Journal of Urology, vol. 31, no. 3, pp. 505-513, 2013.
[11] L. Morelli, S. Guadagni, G. Di Franco, M. Palmeri, G. Di Candio, and F. Mosca, "Da Vinci single site surgical platform in clinical practice: a systematic review," The International Journal of Medical Robotics and Computer Assisted Surgery, vol. 12, no. 4, pp. 724-734, 2016.

[12] B. F. Santos and E. S. Hungness, "Natural orifice translumenal endoscopic surgery: progress in humans since white paper," World Journal of Gastroenterology, vol. 17, no. 13, pp. 16551665, 2011.

[13] P. Wilhelm, S. Axt, P. Storz, S. Wenz, S. Müller, and A. Kirschniak, "Pure natural orifice transluminal endoscopic surgery (NOTES ) with a new elongated, curved transanal endoscopic operation (TEO) device for rectosigmoid resection: a survival study in a porcine model," Techniques in Coloproctology, vol. 20, no. 5, pp. 273-278, 2016.

[14] K. S. Lehmann, J. P. Ritz, A. Wibmer et al., "The German registry for natural orifice translumenal endoscopic surgery," Annals of Surgery, vol. 252, no. 2, pp. 263-270, 2010.

[15] C. Zornig, L. Siemssen, A. Emmermann et al., "NOTES cholecystectomy: matched-pair analysis comparing the transvaginal hybrid and conventional laparoscopic techniques in a series of 216 patients," Surgical Endoscopy, vol. 25, no. 6, pp. 1822-1826, 2011.

[16] K. L. Yung, J. L. K. Cheung, S. W. Chung, S. Singh, and C. K. Yeung, "A single-port robotic platform for laparoscopic surgery with a large central channel for additional instrument," Annals of Biomedical Engineering, vol. 45, no. 9, pp. 2211-2221, 2017.

[17] K. Nakajima, Y. Souma, T. Takahashi et al., "Anatomical measurements to optimize instrumentation for transvaginal surgery," Surgical Endoscopy, vol. 27, no. 6, pp. 2052-2057, 2013.

[18] G. Buess, F. Becerra-Garcia, and M. C. Misra, "Instruments for transluminal laparoscopic surgery or "NOTES"” Minimally Invasive Therapy \& Allied Technologies, vol. 17, no. 6, pp. 331-335, 2008.

[19] A. F. Collins, P. J. Doyle, S. Vilasagar, and G. M. Buchsbaum, "Utility of anterior vaginal wall length measurement in vaginal reconstructive surgery," International Urogynecology Journal, vol. 28, no. 8, pp. 1197-1200, 2017.

[20] H. G. Kenngott, J. J. Wünscher, M. Wagner et al., “OpenHELP (Heidelberg laparoscopy phantom): development of an opensource surgical evaluation and training tool," Surgical Endoscopy, vol. 29, no. 11, pp. 3338-3347, 2015.

[21] G. Vaz, P. Roussouly, E. Berthonnaud, and J. Dimnet, "Sagittal morphology and equilibrium of pelvis and spine," European Spine Journal, vol. 11, no. 1, pp. 80-87, 2002.

[22] R. E. Gutman, H. K. Pannu, G. W. Cundiff, C. F. Melick, S. A. Siddique, and V. L. Handa, "Anatomic relationship between the vaginal apex and the bony architecture of the pelvis: a magnetic resonance imaging evaluation," American Journal of Obstetrics and Gynecology, vol. 192, no. 5, pp. 1544-1548, 2005.

[23] P. Hu, M. Yu, Z. Sun et al., "Analysis of global sagittal postural patterns in asymptomatic Chinese adults," Asian Spine Journal, vol. 10, no. 2, pp. 282-288, 2016.

[24] C. Bonomo, P. Brunetto, L. Fortuna, P. Giannone, S. Graziani, and S. Strazzeri, "A tactile sensor for biomedical applications based on IPMCs," IEEE Sensors Journal, vol. 8, no. 8, pp. 1486-1493, 2008.

[25] Z. Wang, L. Wang, and J. Lee, "Overview on force sensing techniques in robot-assisted minimally invasive laparoscopic surgery," in Proceedings of the 2017 2nd International Conference on Artificial Intelligence: Techniques and Applications (AITA 2017), pp. 239-244, Shenzhen, China, September 2017. 